\title{
Quality improvement in pediatrics: past, present, and future
}

\author{
Stephanie P. Schwartz ${ }^{1}$ and Kyle J. Rehder ${ }^{1,2}$
}

\begin{abstract}
Almost two decades ago, the landmark report "To Err is Human" compelled healthcare to address the large numbers of hospitalized patients experiencing preventable harm. Concurrently, it became clear that the rapidly rising cost of healthcare would be unsustainable in the long-term. As a result, quality improvement methodologies initially rooted in other high-reliability industries have become a primary focus of healthcare. Multiple pediatric studies demonstrate remarkable quality and safety improvements in several domains including handoffs, catheter-associated blood stream infections, and other serious safety events. While both quality improvement and research are datadriven processes, significant differences exist between the two. Research utilizes a hypothesis driven approach to obtain new knowledge while quality improvement often incorporates a cyclic approach to translate existing knowledge into clinical practice. Recent publications have provided guidelines and methods for effectively reporting quality and safety work and improvement implementations. This review examines not only how quality improvement in pediatrics has led to improved outcomes, but also looks to the future of quality improvement in healthcare with focus on education and collaboration to ensure best practice approaches to caring for children.
\end{abstract}

n 1999, the Institute of Medicine's “To Err is Human" report compelled healthcare to address the large numbers of hospitalized patients experiencing preventable harm (1). Concurrently, it became clear that the rapidly rising cost of healthcare would be unsustainable in the long-term. Multiple publications demonstrate the significant morbidity and mortality as well as the added medical costs that accompany a wide variety of preventable errors (2-8), clarifying the need for quality improvement practices in the healthcare setting. Utilizing best practices to eliminate preventable errors such as catheter-associated blood stream infections, ventilator-associated pneumonia, pressure ulcers, and other serious safety events will result in significant cost savings and resource utilization, as well as improved care (9-12). Because of the breadth of its scope, quality improvement has a variety of stakeholders including patients, their families, providers, hospitals, payers, and society as a whole. Unlike many focused research endeavors, quality improvement often crosses different medical subspecialties, departments, and patient populations.

The Institute of Medicine provides overarching guidelines for improving medical care through six aims for improvement (13). These aims include care that is safe (avoiding preventable injuries, reducing medical errors), effective (providing services based on scientific knowledge and clinical guidelines), patient centered (care that is responsive and respectful to individuals), efficient (avoiding wasting time and other resources), timely (reducing wait times and improving patient flow), and equitable (consistent care regardless of patient characteristics and demographics) $(13,14)$. In this review, we will discuss the application of quality improvement to healthcare, highlight the differences between quality improvement and research, discuss several remarkable quality improvements thus far in pediatrics, and conclude by looking to the future of quality improvement in healthcare.

\section{BRINGING QUALITY IMPROVEMENT TO HEALTHCARE}

Rather than focusing on individual responsibility, the Institute of Medicine's "To Err is Human" report attributes the majority of preventable medical errors to systems, processes, and conditions that are poorly designed to lead people away from making mistakes (1). This organizational-based approach directed healthcare leaders to emulate other high-reliability industries as model examples. Industries such as aviation, armed forces, and nuclear power demonstrate low incidences of errors despite high risk and stressful environments mirroring a hospital setting $(15,16)$. The aviation industry learned the need to prioritize safety following early failures and now has a long history of effectively training, measuring, and improving teamwork and safety skills to mitigate error $(17,18)$. Along with the other mentioned high-reliability organizations, aviation created a culture in which safety measures are reliably and effectively practiced (16). While the example that these industries have set can be extrapolated to medicine, such as handoffs occurring in a "sterile cockpit" mimicking aviation practices during takeoffs and landings, it is important to note the difference and unique demands of health care. These differences must be accounted for when undertaking quality improvement interventions in the medical field. To be successful, cultural

${ }^{1}$ Division of Pediatric Critical Care Medicine, Department of Pediatrics, Duke Children's Hospital, Durham, North Carolina; ${ }^{2}$ Physician Quality Officer, Patient Safety Center, Duke University Health System, Durham, North Carolina. Correspondence: Stephanie P. Schwartz (stephanie.permar@duke.edu)

Received 22 April 2016; accepted 24 July 2016; advance online publication 26 October 2016. doi:10.1038/pr.2016.192 
shifts must be tied to solid process improvements that are focused on common elements of preventable errors (16).

Several frameworks have been successfully applied to process improvement efforts in health care, including Model for Improvement $(19,20)$, Lean and Six Sigma Methodologies (21-24), and Failure Mode Effects Analysis (25). As Dr. Kshitij Mistry stated in his paper on handoff communication, regardless of the specific approach to improvement, "the fundamental concept is the same: a system is viewed as a set of interdependent elements acting toward a common goal" (22). The Model for Improvement is a framework that consists of three steps: setting aims to focus improvement, choosing measures to determine if improvement occurs, and testing new ideas to change the current process (19). The Institute for Healthcare Improvement uses this as the framework to guide improvement work. Plan-Do-Study-Act (PDSA) (26-28) is an important tool within the Model for Improvement which allows for rapid cycles to test changes on a smaller scale. During these cycles, knowledge is gained by testing improvements for change and then studying the process (Figure 1). Reflection allows for sustainability or repeating the cycle for new improvements until a gold standard is reached. PDSA is an important iterative process for change but works best within an organized framework (19). The Six Sigma methodology framework began in the manufacturing industry as a performance improvement philosophy and has spread to other industries. Even more so than the Model for Improvement, this data driven approach aims to improve processes by dramatically reducing variability, with an increased focus on reducing defects to less than 3.4 per million outcomes. Six Sigma consists of following five steps: define, measure, analyze, improve, and control $(22,29)$. The "define" phase consists of identifying integral elements of a process and the steps deemed critical to quality. The "measure" phase focuses on understanding the current process. The "analyze" phase pinpoints areas of high variability and attempts to identify potential causes. The "improvement" phase centers on creating solutions to decrease this variability and bridge critical gaps in the current system. Finally, the "control" phase emphasizes efforts that sustain these improvements (22). The Six Sigma framework can be applied to medicine by standardizing activities such as medication delivery and administration

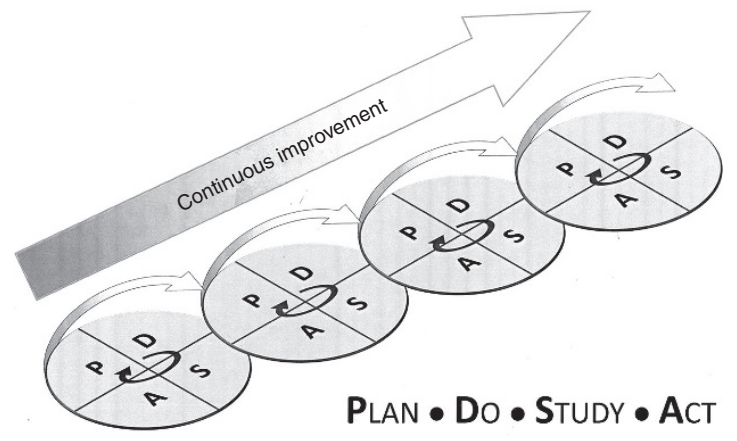

Figure 1. The PDSA cycle. Plan the project objectives, team formation, intervention, and data collection strategy. Do, by carrying out the plan and collecting data. Study the results and summarize learning. Act on any changes needed for the next PDSA cycle. in order to prevent adverse drug events, or standardizing daily rounds to prevent communication breakdowns (30). LEAN is another facet of Six Sigma methodology which focuses on reducing different types of waste, including those centered around materials, time, and staff. For example, as the lean process is applied to rounding, activities that do not add value to the overall goal of efficient and effective rounds such as long travel time between patient rounds, are eliminated $(24,30)$. Also adapted from the manufacturing industry, Failure Mode Effects Analysis is a systematic preemptive strategy to evaluate a process for potential breakdowns, assess the impact of those breakdowns, proactively target solutions, and error-proof the system. Proactive identification and management of potential risks can allow for prevention of medical errors instead of the historical approach of reacting once an event has occurred.

\section{DIFFERENTIATING AND BRIDGING QUALITY IMPROVEMENT AND RESEARCH}

As quality improvement becomes a primary focus of healthcare, it is important to note the differences between quality improvement and traditional research. While both quality improvement and research are data-driven processes, there are significant differences between the two methodologies. Research is focused on generating new knowledge, while quality improvement seeks to improve care by translating existing knowledge into clinical practice. Rather than rigorously testing a single hypothesis with tight variable control, quality improvement incorporates a cyclic approach to generate change in the real world environment. In the setting of quality improvement, the goal is to affect change in real time, necessitating data tracking throughout iterative PDSA cycles. This data is often better presented in run charts and control charts (Figure 2) to help understand variation over time, rather than traditional pre- and postmeasurements (31). Run charts are graphs of ongoing data measurement that help demonstrate patterns which impact improvement (32). Control charts add control limits which define the natural range of variation and can be adjusted after significant process variation. This visual characterization of change over time can more effectively elucidate

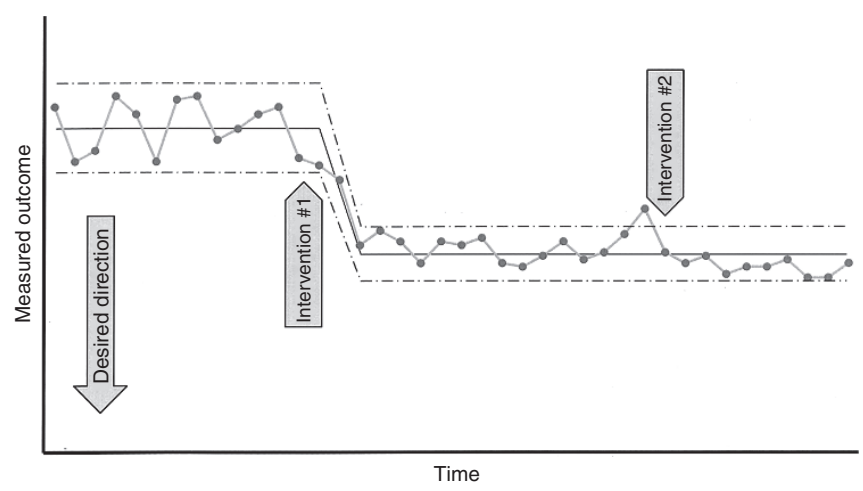

Figure 2. Example of a control chart. The desired metric is traced over time, with interventions annotated to visually demonstrate the effect of those interventions. Upper and lower control limits demonstrated by --Control limits are designated with adjustment after significant process changes. 


\section{Review $\mid$ Schwartz and Rehder}

triggers for improvement, compared to enumerative statistics which require large numbers to demonstrate statistically significant differences following a single intervention, and may fail to differentiate the impact of multiple interventions (33).

Both quality improvement and research are necessary to continue to advance the field of medicine. Patient care is enhanced by combining evidence-based medicine grounded in quality research with optimal healthcare delivery supported by quality improvement strategies. Figure 3 is a graphical representation of reduction in sepsis mortality with multicenter implementation of the evidence based Surviving Sepsis bundle. Sepsis survival correlates with bundle compliance across sites (34). This figure demonstrates an example of the cost of poor quality and inefficient healthcare delivery. When new research yields groundbreaking data which leads to a new standard of care, it is imperative that this information is implemented consistently and in a timely fashion. This figure conceptually demonstrates the reality that it often takes years for new research to become standard of care. The shaded area demonstrates lives lost due to poor implementation of best practices, or patients whose lives may have been saved with optimal application of best practices, highlighting the need for research and quality improvement working together to ensure optimal patient care.

Despite the obvious need for rapid and profound quality improvements in healthcare, the overall pace of change has been slow. The etiology of this delayed pace is complicated and multifactorial, at least partially related to conflicting priorities, unclear methods by which to implement improvement, and financial constraints (16). Due to the inherent differences between research and quality improvement, leaders in the field of quality improvement have argued that the data should be reported differently $(31,35)$. The initial reporting of quality improvement efforts was inconstant and unreliable, causing many publications to be confusing and of limited value, limiting the pace for expansion of quality improvement ideas and methodologies. Recent publications have provided guidelines and methods for effectively reporting quality and safety

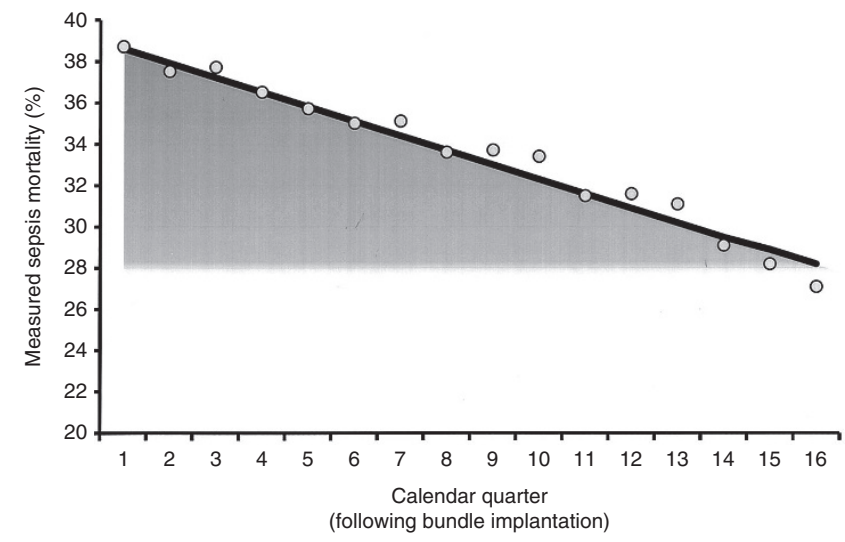

Figure 3. A graphical representation of reduction in sepsis mortality with multicenter implementation of the evidence-based Surviving Sepsis bundle (34). Sepsis survival correlates with bundle compliance across sites. The shaded region represents lives lost due to poor quality, or patients whose lives may have been saved with optimal application of evidence based practices. work and improvement implementations. The first draft publication guidelines for quality improvement were published in 2005 (36) and the Standards for Quality Improvement Reporting Excellence (SQUIRE 1.0) followed in 2008 (37). In 2015, SQUIRE 2.0 was published with revised guidelines (38). These new guidelines are designed to apply to the many varied approaches used for systematically improving quality, safety, and value of healthcare.

Adequately describing the often 'messy' improvement process in peer-reviewed journals can be daunting, and often produces manuscripts very different than traditional research publications. The SQUIRE structure provides a standardized method for publication of quality improvement efforts, highlighting that both the "doing" and the "studying" portions of improvement work are critical for scholarly reporting (38). It includes 18 items divided into four distinct categories (Why did you start?, What did you do?, What did you find?, and What does it mean?) (39). When writing manuscripts, authors should consider all SQUIRE items but recognize that not all items may be included in the final product given that the structure was designed to apply across the many approaches used for systematically improving the quality, safety, and value of healthcare (38). These guidelines direct authors to be transparent, complete, and accurate when reporting all phases of improvement work (38). Sharing of both successes and failures is critical to making the knowledge gained both generalizable and adaptable to other clinical areas and institutions, which may have unique workflows and cultures.

\section{EXAMPLES OF SUCCESSFUL QUALITY IMPROVEMENT IN PEDIATRICS}

Multiple pediatric studies have documented remarkable quality and safety improvements in several domains including catheter-associated blood stream infections, ventilatorassociated pneumonia, patient handoffs, adverse drug events, and other serious safety events $(10,11,16,40-46)$. Review of several of these initiatives highlights the excellent work that has been achieved in improving the quality care of children.

In 2010, the National Association of Children's Hospitals and Related Institutions (NACHRI) group demonstrated that reliably implementing a pediatric specific central line care insertion bundle (checklist) and a new pediatric central line care maintenance bundle can both achieve and sustain significant decreases in pediatric catheter-associated blood stream infection rates in the pediatric intensive care setting (42). This multi-institutional, interrupted time-series design tracked catheter-associated infection rates in 29 pediatric intensive care units (PICUs). Starting with historical control data, this group monitored the number of pediatric catheter-associated blood stream infection rates while promoting compliance with two serially implemented catheter bundles. The intervention reduced the average number of catheter-associated blood stream infection rates by $43 \%$ across 29 PICUs. These statistically significant reductions account for improvement in patient morbidity and mortality as well as decreased costs related to treatment and hospital length of stay, and improved utilization of resources $(12,42)$. While 
catheter-associated blood stream infections in adult intensive care units (ICUs) had been nearly eliminated prior to this study, Miller's study demonstrated a unique difference between pediatric and adult medicine. In contrast to adult ICUs which noted dramatic improvements in infection rates when focusing on the insertion of central lines, the NACHRI study demonstrated that in children, adherence to the maintenance bundle had a much greater impact on the decrease in infection rates when compared to the central line insertion bundle. These differences are likely attributable to different utilization strategies that surround central lines between the adult and pediatric ICUs (42), as well as inherent differences in the patient populations. Physicians training in pediatrics are taught early on that children are not simply "little adults", and therefore it is not surprising that different strategies may be required to optimize care in the pediatric population. The NACHRI study on catheter-associated blood stream infections also stresses the importance on multidisciplinary teams to address quality issues. Numerous pediatric studies demonstrate the strength and value of collaboration between multidisciplinary teams for improvement efforts for pediatric care $(10,23,42,43,47)$.

Patient handoffs, or transitions of care, have been a consistent target for patient safety and quality improvements due to the high percentage of errors that can be attributed to breakdowns in communication $(10,48)$. Patient handoffs have also become increasingly more common as physician trainee work hours have been reduced, potentially negating safety benefits that may result from better rested physicians. The World Health Organization and The Joint Commission recommend a standardized process to reduce handoff related errors $(49,50)$. Transitions of care are particularly high-risk when patients are transferred between units or medical teams, and for medically complex children. Several studies have demonstrated that initiating a structured handoff process between the operating room and the pediatric cardiac ICU following congenital heart disease repair can reduce communication errors and omissions $(22,43,51)$. In 2015, Breuer et al. targeted handover communication in a more generalizable population of all critically ill children being admitted to a multidisciplinary PICU from the operating room. In this study, a standardized handoff protocol was implemented which led to improvement in postoperative handover communication as demonstrated by decreased prevalence of inaccuracies and increased provider attendance and satisfaction with the process. Patient centered outcomes included reduced antibiotic delays and expedited analgesia administration, the latter coupled with improvements in pain scores. This study also demonstrated that the results were sustainable over time (10). Breuer's study also highlights the value of collaboration as multiple disciplines were involved in this handover model including a critical care team comprised of attending physicians, fellows, residents, nurses, and respiratory therapists, a surgical team represented by resident, fellow or attending physicians and an anesthesia team consisting of an attending, nurse anesthetist or member of the house staff.

The Ohio Children's Hospitals' Solutions for Patient Safety (OCHSPS) is one of the best examples of collaboration between hospitals to rapidly and significantly improve the most serious types of harms occurring in hospitalized children (16). OCHSPS represents a group of eight tertiary pediatric referral centers in Ohio. Using a two pronged approach to both change the hospital safety culture while also improving specific processes targeting domains of frequent harm, this group demonstrated a decrease in the serious safety event rate among all hospitals by $55 \%$ (16). These successes were achieved by collectively employing high-reliability methods that focused on safety culture and noncompetitive sharing of outcomes and best practices. Initial work focused on adverse drug events and surgical site infections, but as the leaders recognized the power of collaborative effects, OCHSPS expanded their goal to eliminate all serious harm (16). The serious safety event rate was intentionally selected as measure of success given that these errors strongly impact both providers and patients. This measure highlights the consequences that quality and safety issues have on individual children (16). The goal was to have all members of the hospital able to track the number of children experiencing harm, with the goal of this number eventually becoming zero, instead of a more modest fractional decrease in hospital errors $(16,52)$. In order to achieve this goal, they used a two pronged approach which included developing a culture focused on patient safety and pairing this culture with process improvements led by task forces focused on serious safety events. Hospital leaders made this initiative a priority, private sectors aided in financial support and perhaps most importantly, there was open sharing among the hospitals regarding successes and failures (16). This group, now under the title of 'Solutions for Patient Safety," has expanded beyond the borders of Ohio and now includes more than 80 children's hospitals throughout the country working to eliminate preventable harm in hospitalized children. Pediatrics has a long history of employing collaboration rather than solely competition to improve quality and outcomes. Two other exemplary examples of successful projects in which organized learning, transparency, and the right people have led to improved outcomes for pediatric patients include the Children's Oncology Group, a collaboration of more than 200 pediatric cancer centers which helped increase long-term survival of acute lymphoid leukemia to over $90 \%(53,54)$ and ImproveCareNow which through collaboration of 87 care centers has raised the bar for the standard of care for children with inflammatory bowel disease by significantly increasing remission rates, decreasing steroid use, and improving nutrition (55).

Successful quality improvement initiatives in pediatrics have not only been linked to large, tertiary care centers but also to community hospitals and outpatient clinics. Acute viral bronchiolitis is a high resource utilization pediatric condition due to its prevalence and lack of efficacy of many commonly attempted therapies. In 2006, the American Academy of Pediatrics provided clinical practice guidelines to improve the care of children under the age of two diagnosed with bronchiolitis. These guidelines included limiting unnecessary imaging studies and monitoring, including continuous pulse oximeters, as well as ineffective treatment strategies including bronchodilators, systemic steroids, and antibiotics (56). Following these 
guidelines, multiple academic children's hospitals published their experience in reducing unnecessary care by using standardized recommended guidelines and quality improvement initiatives (57-59). In 2016, Ralston et al. used a voluntary collaborative strategy to disseminate best practices to reduce overuse of unnecessary care in children with bronchiolitis hospitalized outside of major children's hospitals. The collaborative achieved reduction in unnecessary care of bronchiolitis in a majority of community hospital sites including a $68 \%$ reduction in steroid use and a $29 \%$ reduction in bronchodilator use (60). This study is also unique in that the primary focus was on discontinuation of unnecessary therapies which highlights the paucity of quality improvement work on reducing unnecessary care and other waste $(60,61)$. As Ralston hypothesized, it is possible that the sharing of experiences through collaboration is valuable to reduce unnecessary care as it shifts normative behaviors and cultures within a peer group (60).

These endeavors represent a small sample of the quality efforts surrounding the care of children, but are notable for several key elements. While the healthcare delivery of known best practices may be a complex process, many of the solutions to optimizing this delivery are simple, common sense interventions. However, achieving the culture change necessary for success of these interventions takes time and iterative change. Each of these studies demonstrates an understanding of the need for continued improvement with ongoing learning, rather than a single process change. The multidisciplinary approach represented in these studies in both the planning and implementation phases leads to more viable interventions, increases buy-in of new practices, and helps sustainability of the process. Finally, each of these studies aims to not only demonstrate improvement in a process, but to show changes in outcomes with real meaning to patient care.

\section{THE FUTURE OF QUALITY IMPROVEMENT IN PEDIATRICS}

Over the past $15 \mathrm{y}$, quality improvement has become a staple of healthcare. Understanding of quality improvement methodology and participation in the improvement process is now required under both core competencies for physician trainees and maintenance of board certification for practicing physicians. While it is clear that quality improvement efforts in pediatrics has led to improved healthcare delivery and outcomes, there is still much work to be done in order to ensure best practice approaches are applied to the care of children and to eliminate all forms of preventable harm.

As medical research continues to advance at a rapid pace, it will be essential that we couple new knowledge with quality improvement efforts to bring those newly defined best practices to the bedside. At the same time, bold goals and initiatives are necessary to eliminate preventable errors and ensure the highest quality care for all children. In one such initiative, catheter-associated blood stream infections, once thought to be an inevitable event in PICUs, have now been practically eliminated in many units. To continue the success of this and similar work, both financial and staff resources must be allocated to quality and safety efforts.

Quality improvement is tied to the paradigm shift of the US medical system over recent years from pay for service to pay for performance reimbursement with the goal of using payment incentives to recognize and reward high levels of quality and quality improvement (62). Moving forward, reimbursement implications will be an important driver in further raising the profile of quality improvement in academic as well as nonacademic centers. While the majority of initial pay for performance evaluations have focused on the short-term impact of clinical processes such as ophthalmologic exams for diabetic patients, in the future these will likely shift to long-term impact on health outcomes such as complication rates, hospital readmissions, and mortality (63).

The future of quality improvement will move beyond singular endeavors and focus on collaboration among not just individual providers but healthcare organizations as a whole. Despite increasing financial pressures on these organizations which may spark competition, long-term sustainability of this industry will require multi-center partnerships with wide distribution of best practices. Sharing successes and failures will be vital to ensure more rapid improvements across all realms and will lead to optimal care for patients.

Disclosure: The authors have no financial support to disclose. The authors have no conflicts of interest to disclose.

\section{REFERENCES}

1. Kohn LT, Corrigan J, Donaldson MS. To Err Is Human: Building a Safer Health System. Washington, D.C.: National Academy Press; 2000.

2. Aly $\mathrm{H}$, Herson V, Duncan A, et al. Is bloodstream infection preventable among premature infants? A tale of two cities. Pediatrics 2005;115:1513-8.

3. Brilli RJ, Sparling KW, Lake MR, et al. The business case for preventing ventilator-associated pneumonia in pediatric intensive care unit patients. Jt Comm J Qual Patient Saf 2008;34:629-38.

4. Grohskopf LA, Sinkowitz-Cochran RL, Garrett DO, et al.; Pediatric Prevention Network. A national point-prevalence survey of pediatric intensive care unit-acquired infections in the United States. J Pediatr 2002;140: 432-8.

5. Odetola FO, Moler FW, Dechert RE, VanDerElzen K, Chenoweth C. Nosocomial catheter-related bloodstream infections in a pediatric intensive care unit: risk and rates associated with various intravascular technologies. Pediatr Crit Care Med 2003;4:432-6.

6. Urrea M, Pons M, Serra M, Latorre C, Palomeque A. Prospective incidence study of nosocomial infections in a pediatric intensive care unit. Pediatr Infect Dis J 2003;22:490-4.

7. Yogaraj JS, Elward AM, Fraser VJ. Rate, risk factors, and outcomes of nosocomial primary bloodstream infection in pediatric intensive care unit patients. Pediatrics 2002;110:481-5.

8. Costello JM, Morrow DF, Graham DA, Potter-Bynoe G, Sandora TJ, Laussen PC. Systematic intervention to reduce central line-associated bloodstream infection rates in a pediatric cardiac intensive care unit. Pediatrics 2008;121:915-23.

9. Elward AM, Hollenbeak CS, Warren DK, Fraser VJ. Attributable cost of nosocomial primary bloodstream infection in pediatric intensive care unit patients. Pediatrics 2005; 115:868-72.

10. Breuer RK, Taicher B, Turner DA, Cheifetz IM, Rehder KJ. Standardizing postoperative PICU handovers improves handover metrics and patient outcomes. Pediatr Crit Care Med 2015;16:256-63.

11. Brilli RJ, McClead RE Jr, Crandall WV, et al. A comprehensive patient safety program can significantly reduce preventable harm, associated costs, and hospital mortality. J Pediatr 2013;163:1638-45.

12. Nowak JE, Brilli RJ, Lake MR, et al. Reducing catheter-associated bloodstream infections in the pediatric intensive care unit: Business case for quality improvement. Pediatr Crit Care Med 2010;11:579-87.

13. Institute of Medicine, Committee on Quality of Health Care in America. Crossing the Quality Chasm: a New Health System for the 21st Century. Washington, D.C.: National Academy Press; 2001.

14. Wolfe A. Institute of Medicine Report: crossing the quality chasm: a new health care system for the 21st century. Pol Polit Nurs. 2001;2:233-5. 
15. Hudson P. Applying the lessons of high risk industries to health care. Qual Saf Health Care 2003;12 Suppl 1:i7-12.

16. Lyren A, Brilli R, Bird M, Lashutka N, Muething S. Ohio Children's Hospitals' solutions for patient safety: a framework for pediatric patient safety improvement. J Healthc Qual 2016;38:213-22.

17. Catchpole KR, de Leval MR, McEwan A, et al. Patient handover from surgery to intensive care: using Formula 1 pit-stop and aviation models to improve safety and quality. Paediatr Anaesth 2007;17:470-8.

18. Thomas EJ, Sexton JB, Helmreich RL. Translating teamwork behaviours from aviation to healthcare: development of behavioural markers for neonatal resuscitation. Qual Saf Health Care 2004;13 Suppl 1:i57-64.

19. Langley GJ, Moen R, Nolan KM, Nolan TW, Norman CL, Provost LP. The Improvement Guide: a Practical Approach to Enhancing Organizational Performance. John Wiley \& Sons; 2009.

20. Silver SA, Harel Z, McQuillan R, et al. How to begin a quality improvement project. Clin J Am Soc Nephrol 2016;11:893-900.

21. Deblois S, Lepanto L. Lean and Six Sigma in acute care: a systematic review of reviews. Int J Health Care Qual Assur 2016;29:192-208.

22. Mistry KP, Jaggers J, Lodge AJ, et al. Advances in patient safety using six Sigma(R) methodology to improve handoff communication in high-risk patients. In: Henriksen K, Battles JB, Keyes MA, Grady ML, eds. Advances in Patient Safety: New Directions and Alternative Approaches (Vol 3: Performance and Tools). Rockville, MD: Agency for Healthcare Research and Quality (US); 2008.

23. Rehder KJ, Uhl TL, Meliones JN, Turner DA, Smith PB, Mistry KP. Targeted interventions improve shared agreement of daily goals in the pediatric intensive care unit. Pediatr Crit Care Med 2012;13:6-10.

24. Vats A, Goin KH, Villarreal MC, Yilmaz T, Fortenberry JD, Keskinocak P. The impact of a lean rounding process in a pediatric intensive care unit. Crit Care Med 2012;40:608-17.

25. Perks JR, Stanic S, Stern RL, et al. Failure mode and effect analysis for delivery of lung stereotactic body radiation therapy. Int J Radiat Oncol Biol Phys 2012;83:1324-9.

26. Deming WE. Out of the Crisis. Cambridge, MA: Massachusetts Institute of Technology, Center for Advanced Engineering Study; 1986.

27. Deming WE. The New Economics for Industry, Government, Education. Cambridge, MA: Massachusetts Institute of Technology, Center for Advanced Engineering Study; 1993.

28. Moen R. Foundation and History of the PDSA Cycle. Ketchum ID: The W. Edwards Deming Institute; 2015.

29. Plotkin H. Six Sigma: What it is and How to Use it. Boston, MA: Harvard Business School Publishing 1999.

30. Besunder JB, Super DM. Lean Six Sigma: trimming the fat! Effectively managing precious resources. Crit Care Med 2012;40:699-700.

31. Provost LP. Analytical studies: a framework for quality improvement design and analysis. BMJ Qual Saf 2011;20 Suppl 1:i92-6.

32. Institute for Healthcare Improvement. Run Chart Tool, 2012. (http://www. ihi.org/resources/pages/tools/runchart.aspx).

33. Institute for Healthcare Improvement. On Demand: Using Run and Control Charts to Understand Variation. Institute for Healthcare Improvement, 2012. (http://www.ihi.org/education/webtraining/ondemand/run controlcharts/Pages/default.aspx).

34. Levy MM, Rhodes A, Phillips GS, et al. Surviving Sepsis Campaign: association between performance metrics and outcomes in a 7.5-year study. Crit Care Med 2015;43:3-12.

35. Berwick DM. Controlling variation in health care: a consultation from Walter Shewhart. Med Care 1991;29:1212-25.

36. Davidoff F, Batalden P. Toward stronger evidence on quality improvement. Draft publication guidelines: the beginning of a consensus project. Qual Saf Health Care 2005;14:319-25.

37. Davidoff F, Batalden P, Stevens D, Ogrinc G, Mooney S; SQUIRE Development Group. Publication guidelines for quality improvement in health care: evolution of the SQUIRE project. Qual Saf Health Care 2008;17 Suppl 1:i3-9.

38. Ogrinc G, Davies L, Goodman D, Batalden P, Davidoff F, Stevens D. Squire 2.0 (Standards for Quality Improvement Reporting Excellence): revised publication guidelines from a detailed consensus process. Am J Crit Care 2015;24:466-73.

39. Huth EJ, Huth EJ. Writing and publishing in medicine. Baltimore: Williams \& Wilkins; 1999.
40. Sharek PJ, McClead RE Jr, Taketomo C, et al. An intervention to decrease narcotic-related adverse drug events in children's hospitals. Pediatrics 2008;122:e861-6.

41. Bigham MT, Amato R, Bondurrant P, et al. Ventilator-associated pneumonia in the pediatric intensive care unit: characterizing the problem and implementing a sustainable solution. J Pediatr 2009;154:582-587.e2.

42. Miller MR, Griswold M, Harris JM 2nd, et al. Decreasing PICU catheterassociated bloodstream infections: NACHRI's quality transformation efforts. Pediatrics 2010;125:206-13.

43. Chen JG, Wright MC, Smith PB, Jaggers J, Mistry KP. Adaptation of a postoperative handoff communication process for children with heart disease: a quantitative study. Am J Med Qual 2011;26:380-6.

44. Tham E, Calmes HM, Poppy A, et al. Sustaining and spreading the reduction of adverse drug events in a multicenter collaborative. Pediatrics 2011;128:e438-45.

45. Muething SE, Goudie A, Schoettker PJ, et al. Quality improvement initiative to reduce serious safety events and improve patient safety culture. Pediatrics 2012;130:e423-31.

46. Peterson TH, Teman SF, Connors RH. A safety culture transformation: its effects at a children's hospital. J Patient Saf 2012;8:125-30.

47. Mayer CM, Cluff L, Lin WT, et al. Evaluating efforts to optimize TeamSTEPPS implementation in surgical and pediatric intensive care units. Jt Comm J Qual Patient Saf 2011;37:365-74.

48. Bigham MT, Logsdon TR, Manicone PE, et al. Decreasing handoff-related care failures in children's hospitals. Pediatrics 2014;134:e572-9.

49. Joint Commision; Joint Commision International. WHO Collaborating Center for Patient Safety's nine life-saving Patient Safety Solutions. Jt Comm J Qual Patient Saf 2007;33:427-62.

50. The Joint Commission announces the 2009 National Patient Safety Goals and requirements. Jt Comm Perspect 2008;28:1, 11-5.

51. Sheth S, McCarthy E, Kipps AK, et al. Changes in efficiency and safety culture after integration of an I-PASS-supported handoff process. Pediatrics 2016;137:e20150166.

52. Brilli RJ, McClead RE Jr, Davis T, Stoverock L, Rayburn A, Berry JC. The Preventable Harm Index: an effective motivator to facilitate the drive to zero. J Pediatr 2010;157:681-3.

53. Hunger SP, Lu X, Devidas M, et al. Improved survival for children and adolescents with acute lymphoblastic leukemia between 1990 and 2005: a report from the children's oncology group. J Clin Oncol 2012;30: 1663-9.

54. Pui CH, Yang JJ, Hunger SP, et al. Childhood acute lymphoblastic leukemia: progress through collaboration. J Clin Oncol 2015;33:2938-48.

55. Crandall WV, Margolis PA, Kappelman MD, et al.; ImproveCareNow Collaborative. Improved outcomes in a quality improvement collaborative for pediatric inflammatory bowel disease. Pediatrics 2012;129:e1030-41.

56. Diagnosis and management of bronchiolitis. Pediatrics 2006;118:1774-93.

57. Florin TA, Byczkowski T, Ruddy RM, Zorc JJ, Test M, Shah SS. Variation in the management of infants hospitalized for bronchiolitis persists after the 2006 American Academy of Pediatrics bronchiolitis guidelines. J Pediatr 2014;165:786-92.e1.

58. Mittal V, Darnell C, Walsh B, et al. Inpatient bronchiolitis guideline implementation and resource utilization. Pediatrics 2014;133:e730-7.

59. Parikh K, Hall M, Teach SJ. Bronchiolitis management before and after the AAP guidelines. Pediatrics 2014;133:e1-7.

60. Ralston SL, Garber MD, Rice-Conboy E, et al.; Value in Inpatient Pediatrics Network Quality Collaborative for Improving Hospital Compliance with AAP Bronchiolitis Guideline (BQIP). A multicenter collaborative to reduce unnecessary care in inpatient bronchiolitis. Pediatrics 2016;137:.

61. Korenstein D, Falk R, Howell EA, Bishop T, Keyhani S. Overuse of health care services in the United States: an understudied problem. Arch Intern Med 2012;172:171-8.

62. Institute of Medicine, Committee on Redesigning Health Insurance Performance Measures, Payment, and Performance Improvement Programs. Rewarding Provider Performance: Aligning Incentives in Medicare. 2016. Washington, DC: National Academies Press; 2007.

63. Eijkenaar F, Emmert M, Scheppach M, Schöffski O. Effects of pay for performance in health care: a systematic review of systematic reviews. Health Policy 2013;110:115-30. 